\title{
Changes in lifestyles and depressive symptom among patients with chronic diseases during COVID-19 lockdown
}

Wei He

Zhejiang University

\section{Xueyin Zhao}

Zhejiang University

\section{Zhiying Yang}

Zhejiang University

\section{Yan Min}

Stanford University School of Medicine

Yi-Hsuan Wu

Stanford University School of Medicine

\section{Qingcong Kang}

Zhejiang University

\section{Eleanor Frost}

Stanford University School of Medicine

\section{Peng Gao}

Zhejiang University

\section{Yang Yang}

Zhejiang University

\section{Xinyu Chen}

Zhejiang University

\section{Lijin Chen}

Zhejiang University

\section{Ying Lu}

Stanford University School of Medicine

\section{Ann W. Hsing}

Stanford University School of Medicine

Shankuan Zhu ( $\nabla$ zsk@zju.edu.cn )

Zhejiang University 
Keywords: COVID-19, lockdown, China, non-communicable diseases, lifestyle, Depressive symptom Posted Date: March 3rd, 2022

DOI: https://doi.org/10.21203/rs.3.rs-1279952/v1

License: (c) (1) This work is licensed under a Creative Commons Attribution 4.0 International License. Read Full License 


\section{Abstract}

This study aims to investigate the impact of COVID-19 lockdown on lifestyle behaviors and depressive symptom among patients with NCDs. We incorporated a COVID-19 survey to the WELL China cohort, a prospective cohort study with the baseline survey conducted 8-16 months before the COVID-19 outbreak in Hangzhou, China. The COVID-19 survey was carried out to collect information on lifestyle and depressive symptom during lockdown.A total of 3,327 participants were included in the COVID-19 survey, including 2,098 (63.1\%) reported having NCDs at baseline and 1,457 (44\%) without NCDs. The prevalence of current drinkers decreased from $42.9 \%$ before COVID-19 lockdown to $23.7 \%$ during lockdown, current smokers from $15.9-13.5 \%$, and poor sleepers from $23.9-15.3 \%$, while low physical activity increased from 13.4-25.2\%, among participants with NCDs. Participants with NCDs were more likely than those without to have depressive symptom (OR, 1.30; $95 \% \mathrm{Cl}, 1.05-1.61)$, especially among those who need to refill their medication during the COVID-19 lockdown (OR, 1.52; $95 \% \mathrm{Cl}, 1.15-2.02)$.Our findings provide insight into the development of targeted interventions to better prepare patients with NCDs and healthcare system to meet the challenge of future pandemic and lockdown, which is highly likely to happen in the near future.

\section{Introduction}

Since 2020, Coronavirus Disease 2019 (COVID-19) has been diagnosed in over 169 million individuals and caused over 3.53 million deaths worldwide as of June 1 st, $2021,{ }^{1}$ posing an unprecedented challenge to the healthcare system. In China, a total of 110,765 cases have been confirmed, including 4,945 deaths. ${ }^{1}$ Between February and March 2020, China issued a two-month lockdown due to COVID-19 pandemic, mandating all individuals (unless authorized otherwise) to stay at home and stop outdoor activity and social gathering ${ }^{2}$. During the lockdown period, people's lives changed drastically and depressive symptom is likely to be triggered $^{3-5}$.

COVID-19 patients who died were generally older, with a history of noncommunicable diseases (NCDs). ${ }^{6,7}$ Therefore, patients with NCDs may experience more psychological burden during the COVID-19 pandemic or COVID-19 lockdown. It is estimated that 244.5 million people have been diagnosed with hypertension, ${ }^{8}$ $92 \cdot 4$ million with diabetes, ${ }^{9}$ and 94 million with cardiovascular disease in China ${ }^{10}$. These patients generally have a much higher risk of COVID-related morbidity and mortality, and even without COVID infection, their treatment for NCD and quality of life can be affected during the pandemic lockdown due to the impact of health care access and medication refill. Few studies have investigated the impact of COVID-19 lockdown on NCD patients in a community setting.

Within the WELL China cohort that has collected baseline data 8-16 months before COVID-19, we incorporated a survey one month after the COVID-19 lockdown to investigate the impact of COVID-19 lockdown on lifestyle behaviors and depressive symptom among 3,327 cohort members in the Gongshu District, Hangzhou, China. 


\section{Methods}

\section{Study population}

Subjects were a subset of the Wellness Living Laboratory (WELL)-China study. Details of the WELL-China cohort are Described elesewher. ${ }^{13-15}$. Briefly, WELL-China is a population-based cohort for the investigation of well-being in Hangzhou, Zhejiang province, China. In this COVID-19 Study, we surveyed the 4,144 WELL-China participants who completed their baseline survey in Gongshu District, Hangzhou, China, between October 2018 and May 2019, which is 8-16 months before the COVID-19-related nationwide lockdown.

For the COVID-19 survey, we conducted a telephone survey in April 2020, around one month after the lock down, to collect information on lifestyle, mental health and Medical access during the 2-month lockdown (between February and March, 2020; Shelter in Place) period. Of the 4,144 participants at baseline, 3,356 responded to the telephone survey (81\% response rate). We excluded 27 participants who did not report their baseline NCDs conditions, leaving 3,329 for the final analysis.

The WELL-China project was approved by the ethics review board at both Zhejiang University (No. ZGL201507-3) in China and Stanford University (IRB-35020) in USA. All participants provided written informed consent. The follow-up telephone interview was further approved by the ethics review board at Zhejiang University (No. ZGL202004-01). We conducted this study in accordance with the latest revised ethical guidelines of the Declaration of Helsinki.

\section{Baseline Data collection}

During 2018-2019, face-to-face interviews were performed to collect baseline characteristics before the COVID-19 outbreak. Information on noncommunicable diseases (NCDs) was assessed and included selfreported history of diagnosed diabetes, hypertension, cardiovascular disease, cancer, endocrine and metabolic diseases, osteoarthritis, digestive system diseases, respiratory system diseases, mental diseases, urinary system diseases, nervous system diseases, immune diseases, and allergies at baseline. Smoking status and alcohol consumption were categorized as never, former or current. Sleep quality was self-reported as very good, fairly good, fairly bad, or very bad. Eating behavior was categorized into not regular, mostly regular, or very regular. Physical activity was measured using the short version of the International Physical Activity Questionnaire, and were classified into three categories: low; moderate; and vigorous. ${ }^{16}$

\section{Follow-up data collection}

We used telephone follow-up survey to collect the same information on lifestyles and WHO-5 as well as medication refill during the lockdown period. Participants who reported moderate to vigorous physical activity both at baseline and during follow-up survey was defined as maintaining moderate to vigorous physical activity. 


\section{Depressive symptom}

The WHO-Five Well-being Index (WHO-5) was used to assess depressive symptom. The WHO-5 tool is a five-item self-report instrument that assesses well-being (e.g., "I have felt calm and relaxed") over the past two weeks on a 6-point Likert scale ( $0=$ "not present," to $5=$ "constantly present"). Scores on the WHO-5 range from 0 to 25 , and higher scores indicate greater well-being. The WHO-5 has demonstrated good reliability and validity and the ability to identify adults experiencing depression in medical settings in several countries across Africa, Asia, Australia, Europe, North America, and South America ${ }^{17}$.

The five items included: including: (1) "I have felt calm and relaxed", (2) "I have felt cheerful and in good spirits", (3) "I have felt active and vigorous", (4) "My daily life has been filled with things that interest me" and (5) "I woke up feeling fresh and rested". ${ }^{17}$ Depressive symptom was then defined as the participants has answered 0 (at no time) to 1 (some of the time) to any of the five items or if the WHO- 5 raw score below $13^{18}$.

Participants who were classified to have depressive symptom in 2020 during the COVID-19 lockdown but not at baseline (2018-2019) were classified as having incident depressive symptom.

\section{Statistical Analysis}

Chi-square tests were used to assess whether baseline characteristics differed between participants with and without NCDs. McNemar's tests were used to investigate the change in lifestyle and mental health between before and during the COVID19 lockdown.

Univariable and multivariable logistic regressions were used to investigate the association between baseline characteristics and incident depressive symptom during COVID-19 lockdown, among participants with and without NCDs.

We used STATA, version 13 (STATA, College Station, TX), for all of the analyses. All statistical tests were two-sided, and statistical significance was defined as $\mathrm{P}<.05$.

\section{Results}

\section{Baseline characteristics}

Table 1 lists the baseline characteristics of 3,327 study participants by NCDs status. Over three in five $(63 \cdot 1 \%)$ of the participants had NCDs. As shown, participants with NCDs were more likely to be older, widowed, obese, and having worse sleep quality. 
Table 1

Baseline characteristics among 3,327 participants with and without noncommunicable diseases

\begin{tabular}{|c|c|c|c|}
\hline \multirow{3}{*}{ Baseline Characteristics } & \multicolumn{2}{|c|}{ Non-communicable diseases* } & \multirow{3}{*}{$\begin{array}{l}P \text { value* } \\
\mathrm{s}\end{array}$} \\
\hline & No & Yes & \\
\hline & $(n=1,229)$ & $(n=2,098)$ & \\
\hline \multicolumn{3}{|l|}{ Age, yrs } & $<.001$ \\
\hline$<45$ & $377(30.7)$ & $257(12.2)$ & \\
\hline $45-64$ & $688(56.0)$ & 1114(53.1) & \\
\hline$\geq 65$ & 164(13.3) & $727(34.7)$ & \\
\hline \multicolumn{3}{|l|}{ Gender } & 0.912 \\
\hline Male & $461(37.5)$ & 791(37.7) & \\
\hline Female & $768(62.5)$ & 1307(62.3) & \\
\hline \multicolumn{3}{|l|}{ Marital status } & $<.001$ \\
\hline Unmarried & $86(7.0)$ & $58(2.8)$ & . \\
\hline Married & 1080(88.0) & 1882(89.7) & . \\
\hline Divorced & $31(2.5)$ & $57(2.7)$ & \\
\hline Widowed & $30(2.4)$ & $101(4.8)$ & \\
\hline Unknown & 2 & 0 & \\
\hline \multicolumn{3}{|l|}{ Body mass index, $\mathrm{kg} / \mathrm{m}^{2}$} & $<.001$ \\
\hline$<25$ & $906(73.8)$ & $1309(62.4)$ & . \\
\hline $25-29$ & 291(23.7) & 691(33.0) & . \\
\hline$\geq 30$ & $30(2.4)$ & $97(4.6)$ & \\
\hline Unknown & 2 & 1 & \\
\hline \multicolumn{3}{|l|}{ Smoking } & $<.001$ \\
\hline Never & $938(76.5)$ & 1621(77.3) & . \\
\hline Former & $48(3.9)$ & $144(6.9)$ & . \\
\hline \multicolumn{4}{|c|}{ Note: Data were presented as number (\%). } \\
\hline \multicolumn{4}{|c|}{ * $p$ Value was derived from Chi-Square test. } \\
\hline
\end{tabular}




\begin{tabular}{|c|c|c|c|}
\hline \multirow{3}{*}{ Baseline Characteristics } & \multicolumn{2}{|c|}{ Non-communicable diseases* } & \multirow{3}{*}{$\begin{array}{l}P \text { value* } \\
\mathrm{s}\end{array}$} \\
\hline & No & Yes & \\
\hline & $(n=1,229)$ & $(n=2,098)$ & \\
\hline Current & $240(19.6)$ & $333(15.9)$ & . \\
\hline Unknown & 3 & 0 & \\
\hline Drinking & & & 0.005 \\
\hline Never & 722(58.9) & $1157(55.1)$ & \\
\hline Former & $10(0.8)$ & $43(2.0)$ & ${ }^{\circ}$ \\
\hline Current & 494(40.3) & $898(42.8)$ & \\
\hline Unknown & 3 & 0 & \\
\hline Eating irregularly & & & 0.040 \\
\hline No & 1153(94.5) & $2014(96.0)$ & . \\
\hline Yes & $67(5.5)$ & $83(4.0)$ & . \\
\hline Unknown & 9 & 1 & \\
\hline Sleep quality ${ }^{\dagger}$ & & & $<.001$ \\
\hline Very good & $331(27.1)$ & $466(22.2)$ & . \\
\hline Fairly good & $681(55.7)$ & 1132(54.0) & . \\
\hline Fairly bad & $190(15.5)$ & $443(21.1)$ & . \\
\hline Very bad & $20(1.6)$ & $56(2.7)$ & \\
\hline Physical activity & & & $<.001$ \\
\hline Low & $235(20.9)$ & $281(14.0)$ & \\
\hline Moderate & $601(53.4)$ & $1181(58.9)$ & . \\
\hline Vigorous & $289(25.7)$ & $544(27.1)$ & . \\
\hline Unknown & 104 & 92 & . \\
\hline \multicolumn{4}{|c|}{ Note: Data were presented as number (\%). } \\
\hline \multicolumn{4}{|c|}{ * $p$ Value was derived from Chi-Square test. } \\
\hline
\end{tabular}

\section{Lifestyle changes during COVID-19 lockdown}


Figure 1 shows lifestyle changes among study participants by NCD status. Among individuals with NCDs, the prevalence for current alcohol drinkers and current smoker decreased from $4.9-23.7 \%$ and $15.9 \%$ to $13 \cdot 5 \%$, respectively. Participants with self-reported poor sleep quality decreased from $23 \cdot 9 \%$ to $15 \cdot 3 \%$. However, low physical activity increased from $13 \cdot 4 \%$ to $25 \cdot 2 \%$. Similar patterns were found when we restricted our analyses to participants without NCDs.

\section{Changes in prevalence of depressive symptom during COVID-19 lockdown}

Figure 2 shows the prevalence of depressive symptom among individuals with and without NCDs before COVID and during the COVID-19 lockdown. Among individuals with NCDs, the prevalence of depressive symptom during COVID-19 lockdown was $21 \cdot 4 \%(95 \mathrm{Cl}, 19.6 \%$ to $23 \cdot 2 \%)$, significantly higher than the $13.8 \%(95 \% \mathrm{Cl}, 12 \cdot 3-15.3 \%)$ observed at baseline before the COVID-19 lockdown. Similar results were seen for individuals without NCDs.

Table 2 shows the associations between selected characteristics and incident depressive symptom during the lockdown. As shown, younger age and the need to refill medication were associated with a higher risk of having incident depressive symptom during COVID-19 lockdown; whereas maintaining moderate to vigorous physical activity was associated with lower risk of depressive symptom. Further analyses combining NCDs and need to refill medication found that, as compared with no NCDs, NCDs with no need to refill medications was associated with 1.29 (95\% Cls, 1.04-1.58) times increased risk, and NCDs with need to refill medication was associated with $1.48(95 \% \mathrm{Cl}, 1 \cdot 07-2.06)$ times increased risk of having incident depressive symptom during COVID-19 lockdown. 
Table 2

Selected characteristics and their relation with incident depressive symptom during the COVID-19 lockdown $(n=2820)$

\begin{tabular}{|c|c|c|c|c|c|}
\hline & \multicolumn{2}{|c|}{$\begin{array}{l}\text { Incident depressive } \\
\text { symptom } \\
\text { during lockdown }\end{array}$} & \multicolumn{2}{|c|}{ Odds ratios $(95 \%$} & \multirow[b]{2}{*}{$\begin{array}{l}\text { Multivariable } \\
\text { adjusted }+\end{array}$} \\
\hline & $\begin{array}{l}\text { No } \\
(n=2274)\end{array}$ & $\begin{array}{l}\text { Yes } \\
(n=546)\end{array}$ & $s$ & $\begin{array}{l}\text { Age- } \\
\text { adjusted }^{*}\end{array}$ & \\
\hline \multicolumn{6}{|c|}{ Baseline characteristics } \\
\hline \multicolumn{6}{|l|}{ Age, yrs } \\
\hline$<45$ & $373(16.4)$ & $126(23.1)$ & & $\begin{array}{l}1.60(1.26- \\
2.04)\end{array}$ & $\begin{array}{l}1.69(1.31- \\
2.17)\end{array}$ \\
\hline $45-64$ & $1283(56.4)$ & $271(49.6)$ & & 1.00(reference) & 1.00 (reference) \\
\hline$\geq 65$ & $618(27.2)$ & $149(27.3)$ & & $\begin{array}{l}1.14(0.91- \\
1.42)\end{array}$ & $\begin{array}{l}1.06(0.85- \\
1.34)\end{array}$ \\
\hline \multicolumn{6}{|c|}{ Non-communicable disease } \\
\hline No & $859(37.8)$ & 183(33.5) & & 1.00(reference) & 1.00 (reference) \\
\hline Yes & $1415(62.2)$ & $363(66.5)$ & & $\begin{array}{l}1.31(1.07- \\
1.62)\end{array}$ & $\begin{array}{l}1.30(1.05- \\
1.61)\end{array}$ \\
\hline \multicolumn{6}{|l|}{ Gender } \\
\hline Male & $852(37.5)$ & $214(39.2)$ & & 1.00(reference) & - \\
\hline Female & $1422(62.5)$ & $332(60.8)$ & & $\begin{array}{l}0.94(0.78- \\
1.14)\end{array}$ & - \\
\hline \multicolumn{6}{|c|}{ Marital status } \\
\hline Unmarried & 75(3.3) & $23(4.2)$ & & $\begin{array}{l}0.96(0.58- \\
1.59)\end{array}$ & - \\
\hline Married & 2065(90.8) & $484(88.6)$ & & 1.00(reference) & - \\
\hline Divorced & $54(2.4)$ & $12(2.2)$ & & $\begin{array}{l}0.93(0.49- \\
1.75)\end{array}$ & - \\
\hline
\end{tabular}

NOTE: Dashes indicate inapplicable or not included in the model. Women with depressive symptom at baseline were excluded from this analyses.

* Variables were not included in the multivariable model if they were not significantly associated with incident depressive symptom in the univariable models.

† Adjusting all variables listed in this column. 


\section{Incident depressive symptom}

during lockdown

Widowed

80(3.5)

27(4.9)

Odds ratios (95\% Cls)

$1.50(0.95-$

2.37)

Body mass index, $\mathrm{kg} / \mathrm{m}^{2}$

$<25$

1509(66.4) 352(64.6)

1.00(reference) -

$25-29$

683(30.1)

169(31.0)

1.09(0.89-

1.34)

$\geq 30$

$80(3.5)$

24(4.4)

1.26(0.79-

2.02)

Unknown

2

1

Smoking

Never

1743(76.7) 419(76.7)

$33(6.0)$

141(6.2)

$94(17.2)$

$389(17.1)$

1

Unknown

Drinking

Never

Former

Current

Unknown

Eating irregularly

No
1262(55.5) 323(59.2)

$14(2.6)$

$30(1.3)$

$981(43.2)$

1

0

209(38.3)

2185(96.3) 525(96.3)

1.00(reference) -

$1.01(0.68-$

1.49)

1.01(0.79-

1.29)
0

$-$
$-$

1.00(reference) 1.00(reference)

1.91(1.00- $\quad 1.84(0.95-$

3.65)

3.55)

0.83(0.68- $\quad 0.84(0.69-$

1.00) 1.02)

-

1.00(reference) -

NOTE: Dashes indicate inapplicable or not included in the model. Women with depressive symptom at baseline were excluded from this analyses.

* Variables were not included in the multivariable model if they were not significantly associated with incident depressive symptom in the univariable models.

† Adjusting all variables listed in this column. 


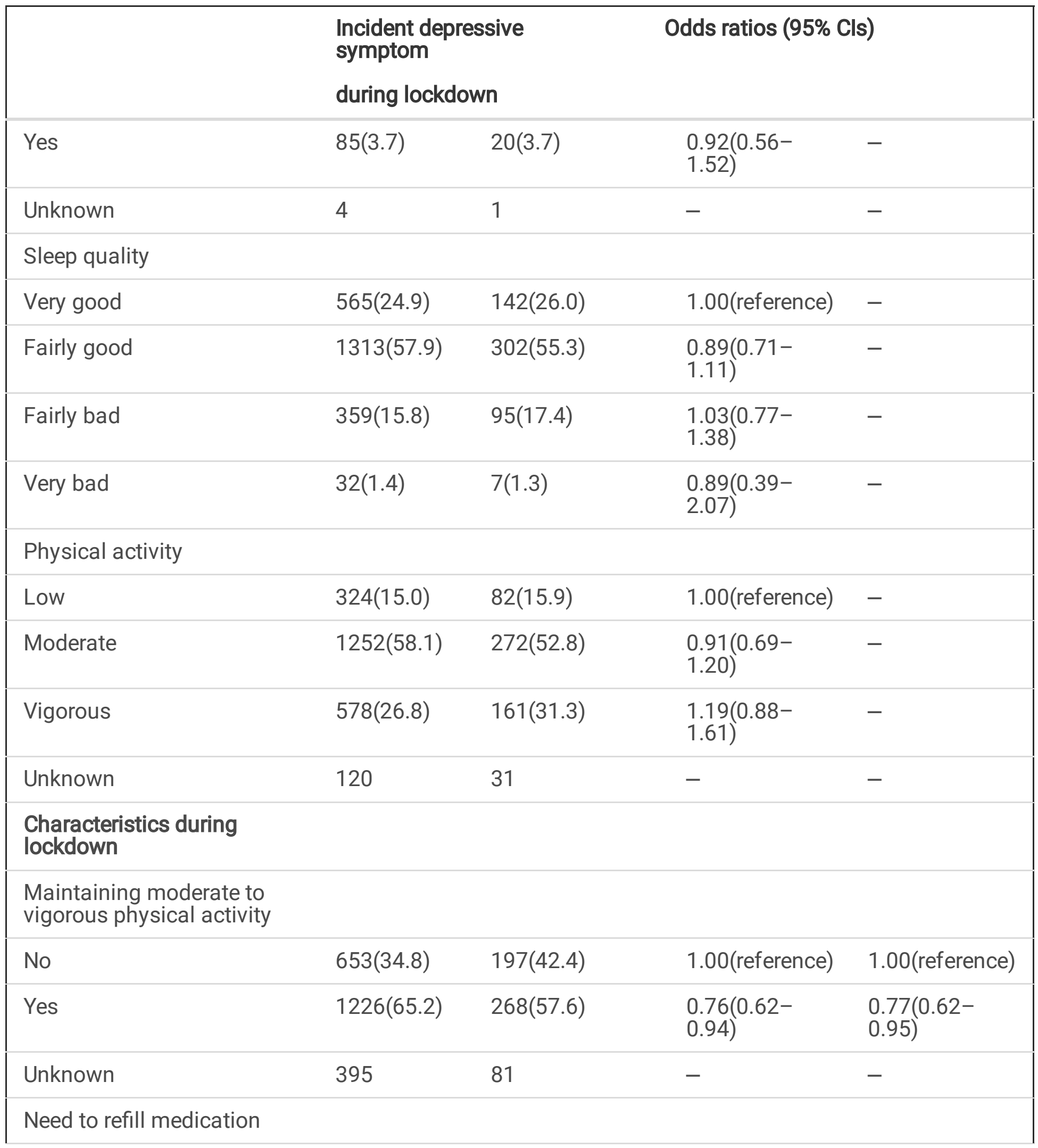

NOTE: Dashes indicate inapplicable or not included in the model. Women with depressive symptom at baseline were excluded from this analyses.

* Variables were not included in the multivariable model if they were not significantly associated with incident depressive symptom in the univariable models.

† Adjusting all variables listed in this column. 


\begin{tabular}{|c|c|c|c|c|}
\hline \multirow[b]{2}{*}{ No } & \multicolumn{2}{|c|}{$\begin{array}{l}\text { Incident depressive } \\
\text { symptom } \\
\text { during lockdown }\end{array}$} & \multicolumn{2}{|c|}{ Odds ratios ( $95 \% \mathrm{Cls}$ ) } \\
\hline & 1708(86.4) & $343(80.5)$ & 1.00(reference) & 1.00 (reference) \\
\hline Yes & 269(13.6) & $83(19.5)$ & $\begin{array}{l}1.62(1.23- \\
2.15)\end{array}$ & $\begin{array}{l}1.52(1.15- \\
2.02)\end{array}$ \\
\hline Unknown & 297 & 120 & - & - \\
\hline \multicolumn{5}{|c|}{$\begin{array}{l}\text { NOTE: Dashes indicate inapplicable or not included in the model. Women with depressive symptom at } \\
\text { baseline were excluded from this analyses. }\end{array}$} \\
\hline \multicolumn{5}{|c|}{$\begin{array}{l}\text { * Variables were not included in the multivariable model if they were not significantly associated with } \\
\text { incident depressive symptom in the univariable models. }\end{array}$} \\
\hline
\end{tabular}

\section{Discussion}

In this large population-based survey, we showed that, during the COVID-19 lockdown in China, the prevalence of low physical activity and depressive symptom increased, while the prevalence of current smoking, drinking, and poor sleep quality decreased. Younger age ( $<45$ years) and the need for medication refill were associated with a higher risk of having incident depressive symptom during the lockdown; whereas maintaining moderate to vigorous physical activity was associated with lower risk of having depressive symptom during COVID-19 lockdown.

Our finding on lifestyle behavior changes during the lockdown period in China provides information on the impact of the COVID-19 pandemic. The lockdown prohibited people from going out and exercising outdoors, likely resulting in the higher prevalence of low physical activity. In contrast, reduced alcohol drinking and smoking, and improved sleep quality are positive changes related to health and well-being during this lockdown period. It is possible that NCDs patients may benefit from the lockdown by reducing risky behaviors and improved sleep. Follow up and further research should investigate the short-term benefits of these behavior changes in patients with NCDs and whether these improvements in healthy lifestyle remain after the lockdown period.

Depressive symptom increased from $13.7 \%$ at baseline (before lockdown) to $20.7 \%$ during lockdown, suggesting an adverse impact of lockdown on mental health. Given the extent of the COVID-19 lockdown worldwide, this high prevalence of depressive symptom suggests that millions of individuals may experience mental health stress during the COVID-19 lockdown. Traditional psychological crisis intervention, e.g., face-to-face contact with qualified mental health professionals, is not likely to be readily available to all who need it. Psychological service system should thus be re-formed to incorporate novel strategies (e.g. online mental health service and telephone-based psychological counselling) to better tackle psychological problem during the COVID-19 epidemic. ${ }^{11,12}$ 
Lack of medical support may also lead to newly emerged depressive symptom among NCDs patients since they were not able to visit the clinic in person for follow up, in particular for imaging or examinations that need in-person service. In addition, in our study, the need to refill medication was associated with a higher risk of having incident depressive symptom during COVID-19 lockdown, suggesting that lack of medication for their chronic conditions may result in concerns and anxiety, leading to depressive symptoms. Collectively, these data suggest the importance of providing adequate clinical support, either through telemedicine or special arrangements, for high-risk individuals, such as patients with chronic diseases during future outbreaks or lockdown to minimize mortality. In addition, mental health of patients with chronic disease needs to be taken into account to minimize anxiety and mental breakdown to minimize the deterioration of the underlying conditions.

The key strength of our study is having longitudinal data on the same individuals before and during COVD-19 in a large population-based study. Limitation of the study should be noted. First, all of our data are self-reported, thereby some misclassification is possible. Second, the follow up survey was carried out one month after the lockdown already completed, thereby some recall issues may contribute to misclassification of information. Fourth, generalizability of our results is limited as access to health care and support vary greatly in different country.

In conclusion, in this community-based study, we found that COVID-19 lockdown significant impact lifestyle and quality of life among individuals with chronic diseases. These results provide the basis for targeted prevention to better prepare NCD patients, community, and healthcare system to meet the challenge in future outbreaks or lockdown.

\section{Declarations}

\section{Acknowledgement}

We would like to thank the participants and community workers of Gongsu, Hangzhou, China for their dedication in this study.

\section{Author contributions statement}

WH, XYZ, MY, AWH, SKZ, designed the study and were responsible for the methodology. WELL-China team carried out the field work investigation. WH and $X Y Z$ analyzed data. WH and $X Y Z$ wrote the manuscript and created the tables and figures. SKZ and AWH supervised all the process of the projects and provided constructive suggestions. WH, XYZ, YM, YHW, ZYY, QCK, EF, PG, YY, XYC, LJC, YL, AWH and SKZ contributed to drafting and critical revision of the manuscript for intellectual content. All authors read and revised the manuscript, and approved the final submitted version.

\section{Competing interests}

The authors declare no conflict of interest. 


\section{Data Availability:}

The datasets generated and analysed during the current study are not publicly available due not publicly available due to protection of participant privacy and confidentiality but are available from the corresponding author on reasonable request.

\section{References}

1. Organization, W. H. COVID-19 Weekly epidemiological update - 1 June 2021. (2021).

2. Zhang, Y., Zhao, Q. \& Hu, B. Community-based prevention and control of COVID-19: Experience from China. Am J Infect Control 48, 716-717, doi:10.1016/j.ajic.2020.03.012 (2020).

3. Ali, I. \& Alharbi, O. M. L. COVID-19: Disease, management, treatment, and social impact. Sci Total Environ 728, 138861, doi:10.1016/j.scitotenv.2020.138861 (2020).

4. Wilder-Smith, A. \& Freedman, D. O. Isolation, quarantine, social distancing and community containment: pivotal role for old-style public health measures in the novel coronavirus (2019-nCoV) outbreak. J Travel Med 27, doi:10.1093/jtm/taaa020 (2020).

5. Park, C. et al. Stress, epigenetics and depression: A systematic review. Neurosci Biobehav Rev 102, 139-152, doi:10.1016/j.neubiorev.2019.04.010 (2019).

6. Wu, C. et al. Risk Factors Associated With Acute Respiratory Distress Syndrome and Death in Patients With Coronavirus Disease 2019 Pneumonia in Wuhan, China. JAMA internal medicine, doi:10.1001/jamainternmed.2020.0994 (2020).

7. Zhou, F. et al. Clinical course and risk factors for mortality of adult inpatients with COVID-19 in Wuhan, China: a retrospective cohort study. Lancet 395, 1054-1062, doi:10.1016/S01406736(20)30566-3 (2020).

8. Wang, Z. et al. Status of Hypertension in China: Results From the China Hypertension Survey, 20122015. Circulation 137, 2344-2356, doi:10.1161/CIRCULATIONAHA.117.032380 (2018).

9. Yang, W. et al. Prevalence of diabetes among men and women in China. The New England journal of medicine 362, 1090-1101, doi:10.1056/NEJMoa0908292 (2010).

10. Liu, S. et al. Burden of Cardiovascular Diseases in China, 1990-2016: Findings From the 2016 Global Burden of Disease Study. JAMA Cardiol 4, 342-352, doi:10.1001/jamacardio.2019.0295 (2019).

11. Liu, S. et al. Online mental health services in China during the COVID-19 outbreak. Lancet Psychiatry 7, e17-e18, doi:10.1016/S2215-0366(20)30077-8 (2020).

12. Bao, Y., Sun, Y., Meng, S., Shi, J. \& Lu, L. 2019-nCoV epidemic: address mental health care to empower society. Lancet 395, e37-e38, doi:10.1016/S0140-6736(20)30309-3 (2020).

13. Min, Y. et al. Sex-specific association between gut microbiome and fat distribution. Nat Commun 10, 2408, doi:10.1038/s41467-019-10440-5 (2019).

14. Min, Y. et al. Cohort Profile: Well living laboratory in China (WELL-China). International journal of epidemiology, doi:10.1093/ije/dyaa283 (2020 (In press)). 
15. Min, Y., Zhao, X., Hsing, A. W. \& Zhu, S. Cohort Profile: WELL living laboratory in China (WELL-China). International journal of epidemiology, doi:10.1093/ije/dyaa283 (2021).

16. Hallal, P. C. \& Victora, C. G. Reliability and validity of the International Physical Activity Questionnaire (IPAQ). Medicine and science in sports and exercise 36,556 , doi:10.1249/01.mss.0000117161.66394.07 (2004).

17. Topp, C. W., Ostergaard, S. D., Sondergaard, S. \& Bech, P. The WHO-5 Well-Being Index: a systematic review of the literature. Psychother Psychosom 84, 167-176, doi:10.1159/000376585 (2015).

18. Halliday, J. A. et al. Validation of the WHO-5 as a first-step screening instrument for depression in adults with diabetes: Results from Diabetes MILES - Australia. Diabetes Res Clin Pract 132, 27-35, doi:10.1016/j.diabres.2017.07.005 (2017).

\section{Figures}

\section{Figure 1}

Lifestyle before and during the nationwide COVID-19 lockdown in Hangzhou, China. * $p<0.05$, for the difference before and during lockdown using McNemar's test. 


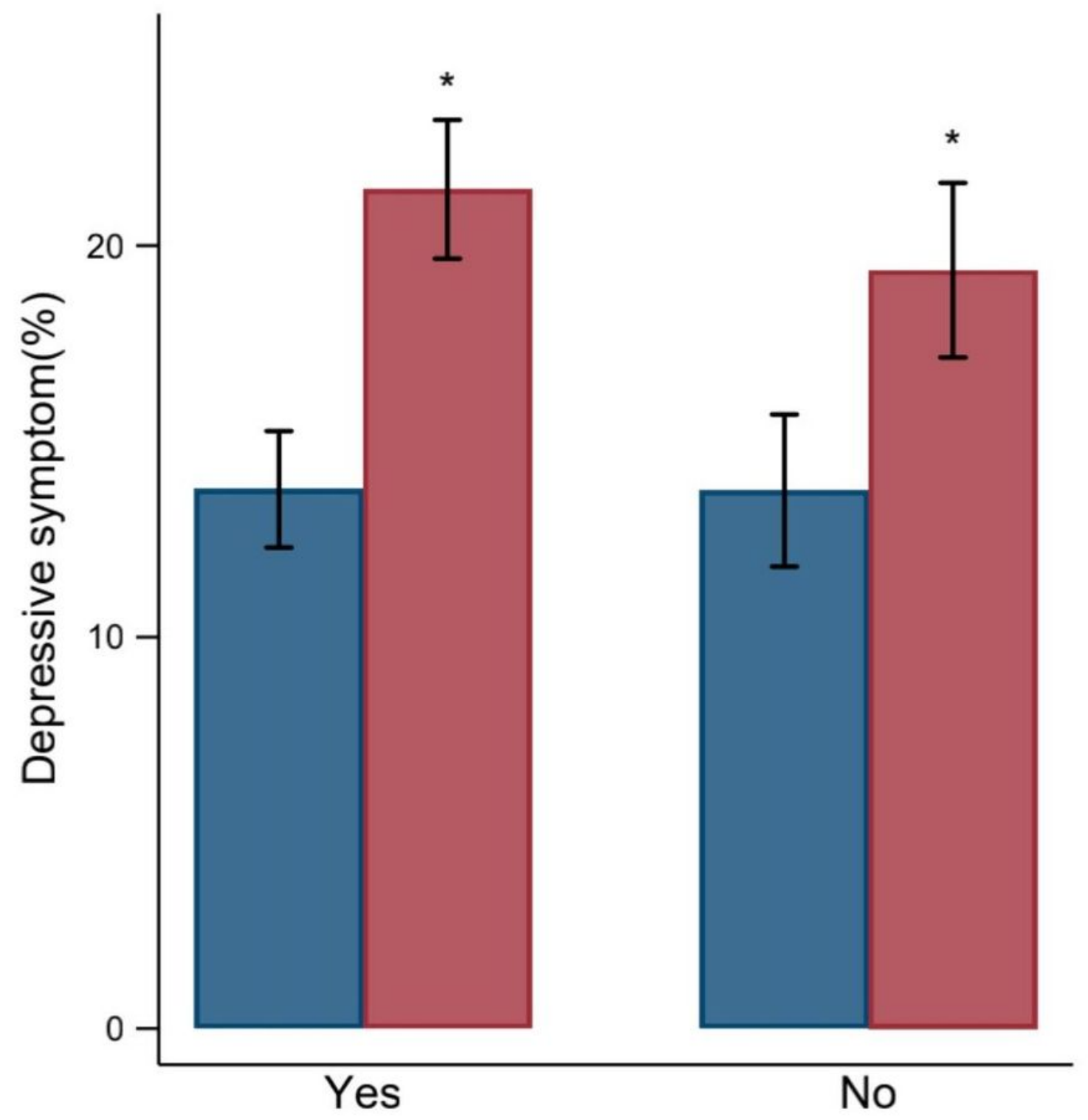

Non-communicable diseases

Figure 2

Depressive symptom before and during the nationwide COVID-19 lockdown in Hangzhou, China. * $p<0.05$, for the difference before and during lockdown using McNemar's test. 